\title{
RECURSOS EDUCATIVOS DIGITALES
}

\author{
Claudia Piriz - Verónica Lazzarini \\ claudiapirizcotignola@,hotmail.es - veromat1981@,hotmail.com \\ Liceo n`57 - Liceo El Pinar n² - Uruguay
}

Tema: Uso de tecnologías

Modalidad: $\mathrm{T}$

Nivel educativo: Medio

Palabras claves: Recursos digitales, TIC, Interactivo

Se pretende compartir actividades interactivas y juegos que han motivado a los alumnos de Ciclo Básico, como forma de fortalecer el aprendizaje a través del uso de la tecnología. Utilizando la plataforma Crea2 de Ceibal, se insertan algunos recursos educativos digitales de Matemática que existen en la red; tales como las actividades interactivas, videos, puzles y juegos; los cuales se dejan a disposición de los docentes, con el objetivo de que puedan explorar, investigar qué contenidos y/o habilidades permiten abordar, discutir las fortalezas y debilidades de cada recurso, analizar posibles inconvenientes así pensamos entre todos cómo se pueden solucionar.

A modo de ejemplo agregamos dos actividades:

http://www.arcademics.com/games/spider-match/spider-match.html

http://www.primaria.librosvivos.net/archivosCMS/3/3/16/usuarios/103294/9/6EP_Mat ud10_ResuelveProblemas/frame_prim.swf

Se mostrarán algunas páginas de internet que permiten crear actividades y presentaciones, tales como http://www.educaplay.com/ y http://www.powtoon.com/home/g/es/.

Introducción:

¿Por qué elegimos trabajar con Recursos Educativos Digitales?

El avance de la tecnología ha modificado la comunicación y la forma de obtener la información, lo cual influye directamente en las nuevas generaciones y sus formas de vincularse al conocimiento. Por ese motivo, sentimos la necesidad de encontrar estrategias que se adecuen al cambio, con el objetivo de motivar a los alumnos, enriquecer la práctica de aula y potenciar el aprendizaje. Comenzamos a investigar qué recursos ofrece la web, seleccionando aquellos que consideramos adecuados para abordar en el aula. Y los propusimos en nuestras clases. En esta instancia, queremos compartir la experiencia de incluir los recursos educativos digitales en la práctica de aula.

¿Cómo desarrollaremos el taller? 
Luego de presentarnos, realizaremos un "recorrido" por la plataforma Crea 2, explicando cómo se agregan las herramientas externas (es una de las formas que permiten agregar los recursos educativos digitales para que accedan los alumnos) y cómo crear el espacio para que suban las capturas de pantalla de las actividades realizadas. Desde dicha plataforma, compartiremos una presentación creada en la página http://www.powtoon.com/home/g/es/, con el fin de explicar brevemente (mediante uno de los recursos que propondremos como herramienta de trabajo en el aula) qué son los recursos educativos digitales y cómo los abordaremos desde el taller. Profundizaremos en la página powtoon, compartiendo ejemplos de actividades de matemática que han sido diseñadas utilizando dicho recurso. Agregamos otro enlace de creación de actividades: http://www.educaplay.com/. En principio, se mostrará el procedimiento para registrarse y la forma de acceder a las actividades creadas por otros usuarios, luego, profundizamos en el diseño de actividades. Para que la experiencia sea enriquecedora, nos parece fundamental que los docentes experimenten, que pasen del rol de profesores a sentir que están en el lugar de los alumnos. Es por eso que, en esta instancia generamos un primer espacio para explorar el enlace mencionado, con la siguiente consigna:

$\underline{\text { Primera consiga de trabajo: (15 minutos) }}$

Reunirse en equipos de 2 integrantes, registrarse uno de ellos en la página http://www.educaplay.com/, explorar las actividades que están creadas y diseñar una de cualquier tema de Matemática; la cual compartiremos en una puesta en común.

Se realiza la puesta en común de las actividades creadas por cada equipo.

Presentamos otras páginas donde se pueden crear actividades, tales como sopas de letras, crucigramas y puzles.

$\underline{\text { Segunda consigna de trabajo: (10 minutos) }}$

Exploren las siguientes páginas:

https://www.educima.com/wordsearch/spa/ (Generador de sopas de letras)

https:/www.educima.com/crosswordgenerator/spa/ (Generador de crucigramas) 
http:/www.pekegifs.com/puzzles/generadorpuzzles.htm (Generador de puzles)

Luego, reflexionen sobre los materiales a partir de las siguientes preguntas:

a) ¿Estás páginas son útiles para diseñar actividades que motiven a los alumnos?

b) ¿Estos generadores, se pueden utilizar para promover el aprendizaje?

c) ¿En qué niveles se puede utilizar?

d) ¿Qué contenidos de Matemática se puede abordar utilizando estos generadores? (pensar en por lo menos un contenido para cada generador).

Se realiza un intercambio de respuestas de todos los equipos.

Compartiremos otros recursos educativos digitales que están a disposición en la web: Acertijos:

http:/www.plastelina.net/game1.html

Tres en raya:

http:/www.primaria.librosvivos.net/archivosCMS/3/3/16/usuarios/103294/9/6EP Mat ud10 ResuelveProblemas/frame prim.swf

Adición de números enteros:

http:/www.arcademics.com/games/spider-match/spider-match.html

Multiplicación de números enteros:

http:/www.arcademics.com/games/integer-warp/integer-warp.html

Cuadrados mágicos:

http:/contenidos.educarex.es/sama/2002/UsaElCoco/calculo/cuadrados/cuadrados.htm

Múltiplos y divisores:

http:/www.matemath.com/juegos1.php?cadena=1-2

Tercera consigna de trabajo: (20 minutos)

Exploren todos los enlaces.

Luego, seleccionen uno de los recursos y completen la siguiente ficha: 


\begin{tabular}{|l|l|l|}
\hline Recurso seleccionado & \\
¿Consideran que promueve el & \\
aprendizaje? \\
¿Qué objetivos se pueden plantear con
\end{tabular}

A partir de la puesta en común de las fichas, se genera espacio de discusión.

Cuarta consigna de trabajo: (30 minutos)

Investiguen los siguientes recursos educativos digitales:

http://www.primaria.librosvivos.net/archivosCMS/3/3/16/usuarios/103294/9/6EP Mate cas ud8_Porcentaje/frame_prim.swf

http://escritorioalumnos.educ.ar/datos/recursos/juegos/leyenda-ajedrez/M016 intro.swf

http://www.ceibal.edu.uy//elp/ODEA_primos_Lazzarini_NLPMOU.elp/index.html

http://www.educaplay.com/es/recursoseducativos/1549722/divisibilidad.htm

https://www.youtube.com/watch?v=b2qsDRIFyb0

http://www.primaria.librosvivos.net/archivosCMS/3/3/16/usuarios/103294/9/6EP_Mat_cas_a ct_ud5_198/frame_prim.swf 
https://3553d525fee3cad6a1caf9675a3cd9b2ccf9d123.googledrive.com/host/OB1FNvXTiebd0 dnZ5dTg3T1B5NUk/index.html

https://4db17b18d17859037465072dce4872ba7a52d75f.googledrive.com/host/0BzPW2 c9gzoAX21GYUhhQVJ1WjA/index.html

https:/43ff0dd596bc9e3794111 e91 fdbad75d5001e845.googledrive.com/host/0B-

tZet05JhxAOGRUYWRVUnVIVGc/actividad 10 recapitulacin.html

https://ea5ac1bbb59a3794db3dc350592895ad3ab9d98c.googledrive.com/host/0B0CG9 Z6xUDnAZ3ctX19OajNANA/ejercicios con potencias.html

http:/www.santillanaenred.cl/hipertextos/2009/matematica $8 /$ recursos/la casa/es carcas $\underline{\text { a.html }}$

http:/www.primaria.librosvivos.net/archivosCMS/3/3/16/usuarios/103294/9/6EP Mat es ud12 ResuelveProblemas/frame prim.swf

http://www.disfrutalasmatematicas.com/juegos/4-en-linea.html

http://genmagic.org/mates1/ra1c.swf

https://www.powtoon.com/show/bOlvhFS7XOO/actividad-final/

Luego, elijan un enlace que consideren adecuado para abordar en clase y realicen un borrador de planificación, completando la siguiente ficha:

\begin{tabular}{|l|l|}
\hline Enlace del recurso elegido: & \\
\hline Nivel: & \\
\hline Objetivos: & \\
\hline Contenidos a abordar: & \\
\hline Tiempo destinado al recurso: & \\
\hline Modalidad de trabajo: & \\
\hline Posibles emergentes: & \\
\hline Evaluación & \multicolumn{2}{|c|}{ Preguntas } \\
\hline \multicolumn{2}{|c|}{ ¿Creen que los recursos educativos digitales favorecen el aprendizaje? ¿Por qué? } \\
\hline
\end{tabular}




\begin{tabular}{|l|}
\hline ¿Estos recursos complementan el trabajo de aula o sustituyen alguna otra herramienta \\
o forma de trabajo? \\
\hline $\begin{array}{l}\text { ¿Trabajar con recursos educativos digitales, favorece a los estudiantes con dificultades } \\
\text { de aprendizaje? ¿Por qué? }\end{array}$ \\
\hline ¿Utilizarían alguno de estos recursos en la práctica de aula? ¿Por qué? \\
\hline ¿Qué ventajas y desventajas identifican en los recursos educativos digitales? \\
\hline
\end{tabular}

Generamos el espacio para el debate a partir de las preguntas planteadas.

Finalizamos el taller con la entrega de una evaluación sobre nuestro trabajo:

Agradecemos que completen la siguiente encuesta, sus comentarios nos ayudarán a mejorar la implementación de futuros talleres.

\begin{tabular}{|c|c|c|c|}
\hline & $\mathrm{Si}$ & Más o menos & No \\
\hline \multicolumn{4}{|l|}{ ¿El taller cumplió con tus expectativas? } \\
\hline \multicolumn{4}{|l|}{ ¿Te aportó nuevas ideas? } \\
\hline \multicolumn{4}{|l|}{$\begin{array}{l}\text { ¿Crees que lo trabajado enriquecerá tus prácticas de } \\
\text { aula? }\end{array}$} \\
\hline \multicolumn{4}{|l|}{$\begin{array}{l}\text { ¿Te motivó a integrar los recursos educativos } \\
\text { digitales en tus clases? }\end{array}$} \\
\hline \multicolumn{4}{|l|}{ ¿Las instancias de discusión fueron productivas? } \\
\hline \multicolumn{4}{|l|}{$\begin{array}{l}\text { ¿Necesitas más tiempo para profundizar en este } \\
\text { tema? }\end{array}$} \\
\hline \multicolumn{4}{|c|}{ 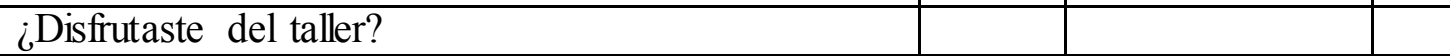 } \\
\hline \multicolumn{4}{|c|}{ ¿Has trabajado con recursos educativos digitales? } \\
\hline \multicolumn{4}{|c|}{ ¿Qué te aportó el taller? } \\
\hline \multicolumn{4}{|c|}{ ¿Qué cambiarías para mejorar nuestra forma de trabajo? } \\
\hline \multicolumn{4}{|c|}{ ¿Recomendaría este taller a otras personas? } \\
\hline Comentarios & & & \\
\hline
\end{tabular}


Luego, agradecemos la participación y el interés por el taller. Esperamos que los recursos compartidos sean de utilidad para los docentes.

\section{Bibliografía:}

Area Moreira, M. (2003) "De los webs educativos al material didáctico web". Comunicación y Pedagogía, n¹88, p. 32-38.

Barroso, J. Cabrero, J. (2013) “Nuevos escenarios digitales”. Madrid, España - Pirámide.

Carneiro, R. Toscano, J. Díaz, T. (2009) "Los desafíos de las TIC para el cambio educativo". Organización de Estados Iberoamericanos. Madrid, España - Fundación Santillana.

EDUTEC. (2009) "Ventajas del uso de las TICs en el proceso de enseñanza-aprendizaje desde la óptica de los docentes universitarios españoles". Revista electrónica de Tecnología Educativa.

Murillo, J. Krichesky G. (2012). "El proceso del cambio escolar. Una guía para impulsar y sostener la mejora de las Escuelas". Revista Iberoamericana sobre Calidad, Eficacia y Cambio en Educación. Volumen 10, Número 1.

UNESCO (2008). "Estándares de competencias en TIC para docentes". Londres, disponible en: http://www.eduteka.org/EstandaresDocentesUnesco.php. 